\title{
Dual-emitting dot-in-bulk CdSe/CdS nanocrystals with highly emissive core- and shell-based trions sharing the same resident electron
}

Valerio Pinchetti ${ }^{*}$, Elena V. Shornikova ${ }^{2}$, Gang Qiang ${ }^{2}$, Wan Ki Bae ${ }^{4}$, Francesco Meinardi ${ }^{1}$, Scott A. Crooker $^{5}$, Dmitri R. Yakovlev ${ }^{2,3}$, Manfred Bayer ${ }^{2,3}$, Victor I. Klimov ${ }^{4 *}$ and Sergio Brovelli ${ }^{*}$

${ }^{1}$ Dipartimento di Scienza dei Materiali, Università degli studi di Milano-Bicocca, via Roberto Cozzi 55, I-20125 Milano, Italy

${ }^{2}$ Experimentelle Physik 2, Technische Universität Dortmund, 44227 Dortmund, Germany

${ }^{3}$ Ioffe Institute, Russian Academy of Sciences, 194021 St. Petersburg, Russia

${ }^{4}$ Chemistry Division, Los Alamos National Laboratory, Los Alamos, New Mexico 87545, United States

5 National High Magnetic Field Laboratory, Los Alamos National Laboratory, Los Alamos, New Mexico 87545, United States

*Corresponding authors: valerio.pinchetti@unimib.it, klimov@lanl.gov, sergio.brovelli@unimib.it 


\section{Methods}

\section{Synthesis of dot-in-bulk (DiB) nanocrystals (NCs) and structural characterization}

$\mathrm{CdSe} / \mathrm{CdS} \mathrm{DiB}$ NCs were synthesized following the procedure described in ref. ${ }^{1}$. Briefly, zincblende CdSe NCs were prepared using previously reported methods ${ }^{2}$. For the synthesis of CdSe (radius $1.5 \mathrm{~nm}$ ) $/ \mathrm{CdS} \mathrm{NCs}, 2 \times 10^{-7} \mathrm{~mol}$ of CdSe NCs (purified twice) dispersed in $10 \mathrm{~mL}$ of 1-octadecene (ODE) were loaded into a $100 \mathrm{~mL}$ flask, degassed at $110^{\circ} \mathrm{C}$ for $1 \mathrm{~h}$. The flask was filled with Ar and heated up to $300{ }^{\circ} \mathrm{C}$ for $\mathrm{CdS}$ shell growth. A $0.2 \mathrm{mmol}$ sample of Cd-oleate and $0.2 \mathrm{mmol}$ of 1-dodecanethiol were added slowly $(0.1 \mathrm{mmol} / \mathrm{min})$ and the reaction was maintained at the elevated temperature for $30 \mathrm{~min}$ to form a thin CdS buffer layer ( $\sim 3$ monolayers) on top of CdSe cores. For further CdS shell growth, a mixed solution of Cd-oleate and trioctylphospine-sulfur $(0.5 \mathrm{M} / 0.5 \mathrm{M})$ in ODE was continuously added at a rate of $1 \mathrm{mmol} /$ hour at $300^{\circ} \mathrm{C}$. After the injection of precursors was completed, the reaction products were cooled to room temperature and purified repeatedly by a precipitation-and-redispersion method. The final products were dispersed in hexane for further characterization. Transmission electron microscopy (TEM) images were obtained using a JEOL 2010 transmission electron microscope. This procedure produced $\mathrm{DiB}$ NCs with the $1.5 \mathrm{~nm}$ CdSe core radius and the $8.5 \mathrm{~nm}$ shell thickness.

\section{Spectroscopic studies}

Steady-state and time-resolved photoluminescence (PL) spectra were obtained using excitation from a pulsed diode laser with the 3.06-eV photon energy (Edinburgh Inst. EPL 405, 40 ps pulse width) and a variable pulse repetition rate. The DiB NC samples were prepared as drop-cast films on glass substrates. For magneto-optical measurements, they were placed in a variable-temperature insert of a closed-cycle helium cryostat $(T=3.5-300 \mathrm{~K})$. The emitted light was coupled into a $600 \mu \mathrm{m}$ optical fiber and the PL signal was spectrally resolved with a TM-C10083CA Hamamatsu Mini-Spectrometer. The PL dynamics were studied with a Hamamatsu R943-02 time-correlated single-photon counting unit coupled to an Oriel Instruments Cornerstone 260 monochromator.

For PL studies in high pulsed magnetic fields, the samples were mounted onto a custom fiber-coupled probe that, in turn, was loaded into a helium bath cryostat with a long tail extending into the bore of a 65 T-class pulsed magnet. Light was directed to and collected from the sample using a single $600 \mu \mathrm{m}$ diameter multimode optical fiber. Thin film circular polarizers were used to select emitted light with a certain handedness of circular polarization. Full optical spectra were acquired every 1 ms continuously throughout the magnet pulse ( $\sim 50 \mathrm{~ms}$ duration) using a fast CCD camera (Princeton Instruments Blaze). To switch between $\sigma^{+}$and $\sigma^{-}$circular polarizations, we switched current direction in the magnet.

Magneto-optical measurements in a DC magnetic field $(B)$ up to $15 \mathrm{~T}$ were performed using a magnetooptical setup based on a cryostat with a superconducting single-coil solenoid. An NC sample was kept in helium exchange gas at a $4.2 \mathrm{~K}$ temperature. Direct optical access to the sample was achieved using the top window. The $B$ field was applied in the Faraday geometry wherein its direction was parallel to a wave vector of collected PL. The PL signal was dispersed using a $0.55-\mathrm{m}$ spectrometer and detected by a liquid-nitrogen-cooled CCD or an avalanche Si-photodiode connected to a conventional time-correlated single-photon counting system. PL was excited with a pulsed diode laser (photon energy $3.06 \mathrm{eV}$, pulse duration $50 \mathrm{ps}$ ). The temporal resolution of this experiment was $100 \mathrm{ps}$. 
Supporting Figure S1 - X-Ray Diffraction Pattern of CdSe/CdS dot-in-bulk (DiB) nanocrystals (NCs).

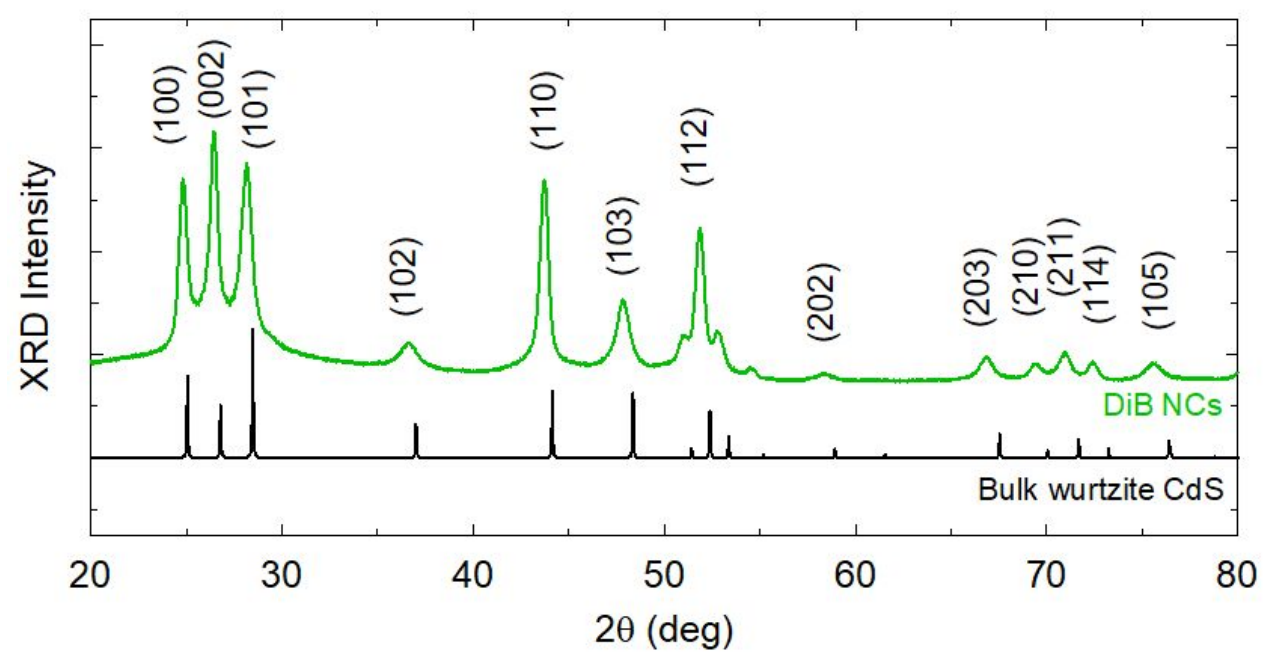

Supporting Figure S1 - The X-ray diffraction pattern of CdSe/CdS DiB NCs (green line) compared to the pattern of bulk CdS in wurtzite crystal structure (black line). 
Supporting Figure S2 - Transmission Electron Microscopy Image of CdSe/CdS DiB NCs.

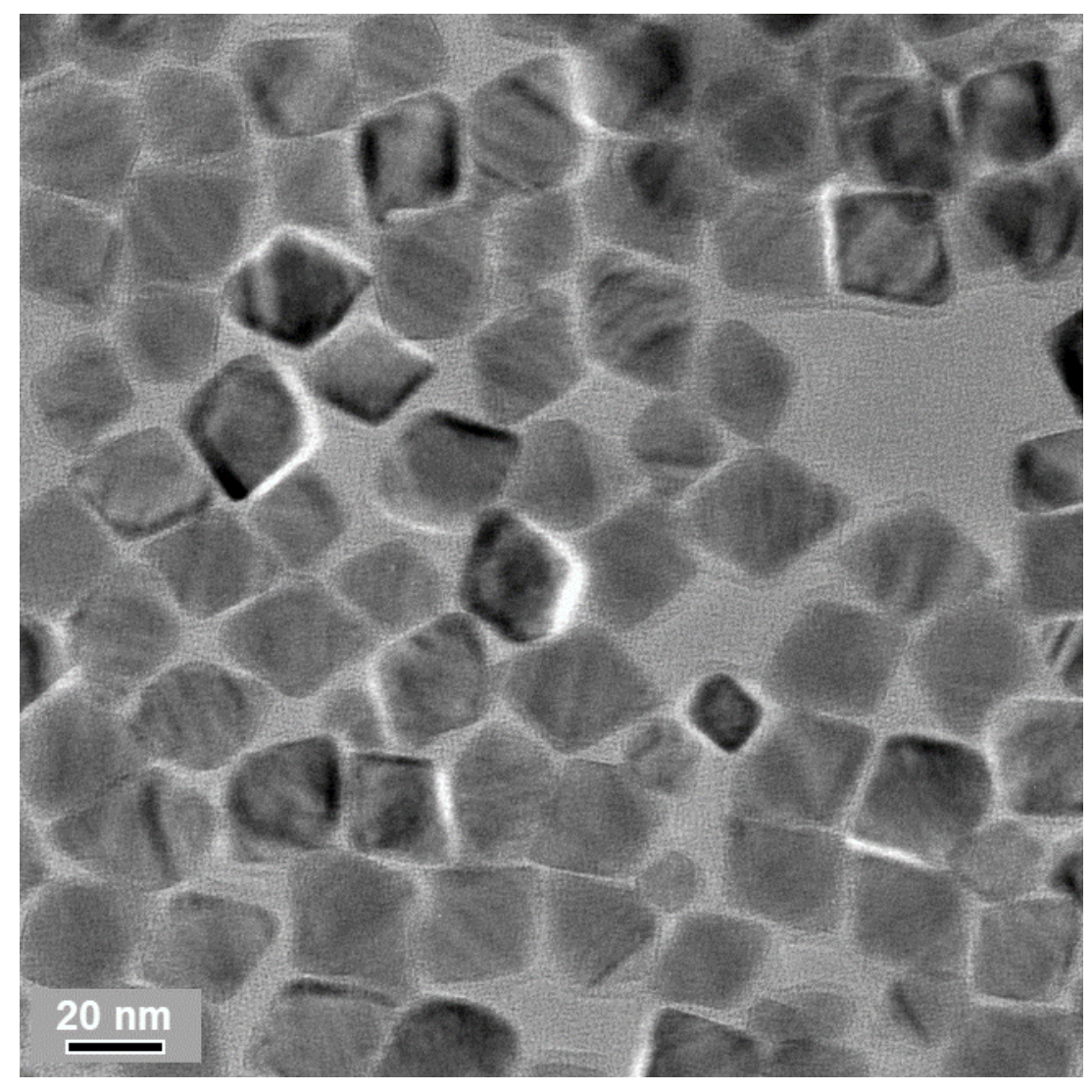

Supporting Figure S2 - Transmission electron microscopy (TEM) image of CdSe/CdS DiB NCs indicates a non-spherical shape due to the ultra-thick CdS shell (thickness of $\sim 8.5 \mathrm{~nm}$ ). 
Supporting Figure S3 - Photoluminescence (PL) decay in DiB NCs as a function of Magnetic Fields.
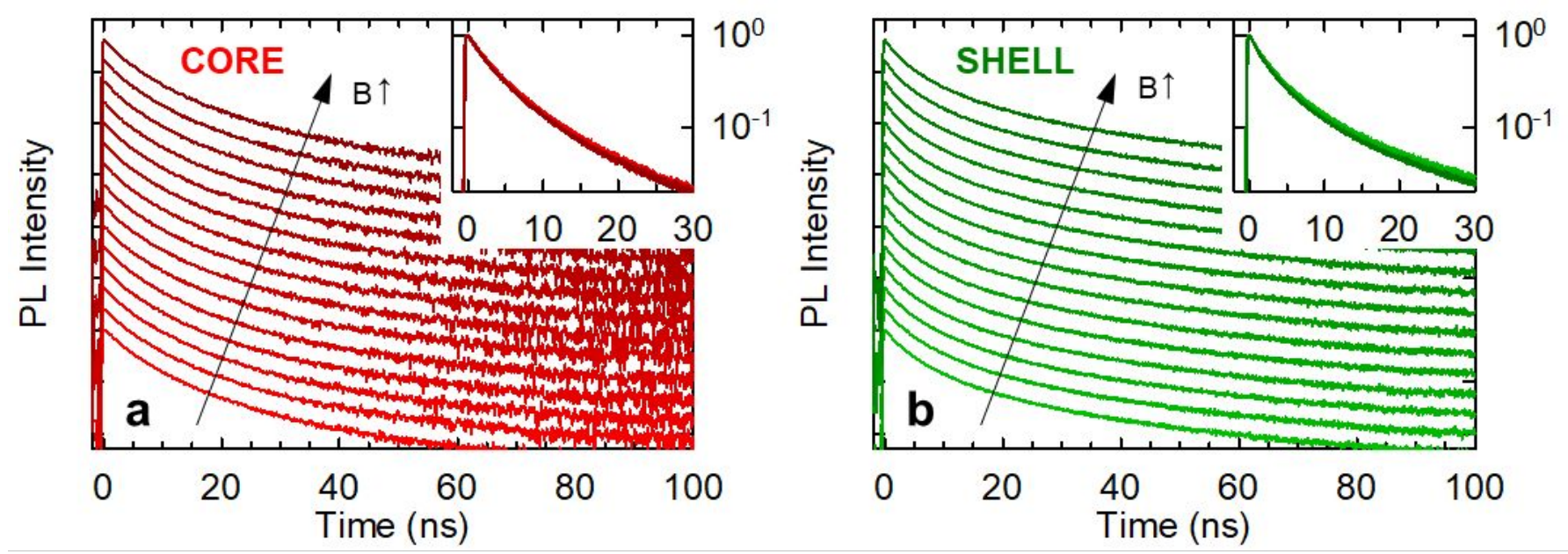

Supporting Figure S3 - (a) Core and (b) shell PL decays at increasing $B$ (from 1 to 15 T with 1 T steps) and $T=4.2 \mathrm{~K}$. The PL traces are offset vertically for clarity. Insets: the expanded view of the first $30 \mathrm{~ns}$ of the same data; the PL traces are superimposed to highlight that no changes occur upon application of magnetic field. For the core PL decay, $k_{\text {rad }}(1 \mathrm{~T})=0.23 \mathrm{~ns}^{-1}$ and $k_{\text {rad }}(15 \mathrm{~T})=0.25 \mathrm{~ns}^{-1}$, whereas for the shell PL decay, $k_{\text {rad }}(1 \mathrm{~T})=0.26 \mathrm{~ns}^{-1}$ and $k_{\text {rad }}(15 \mathrm{~T})=0.29 \mathrm{~ns}^{-1}$. 
Supporting Figure S4 - Schematic depiction of the high-field magneto-optical experiment.

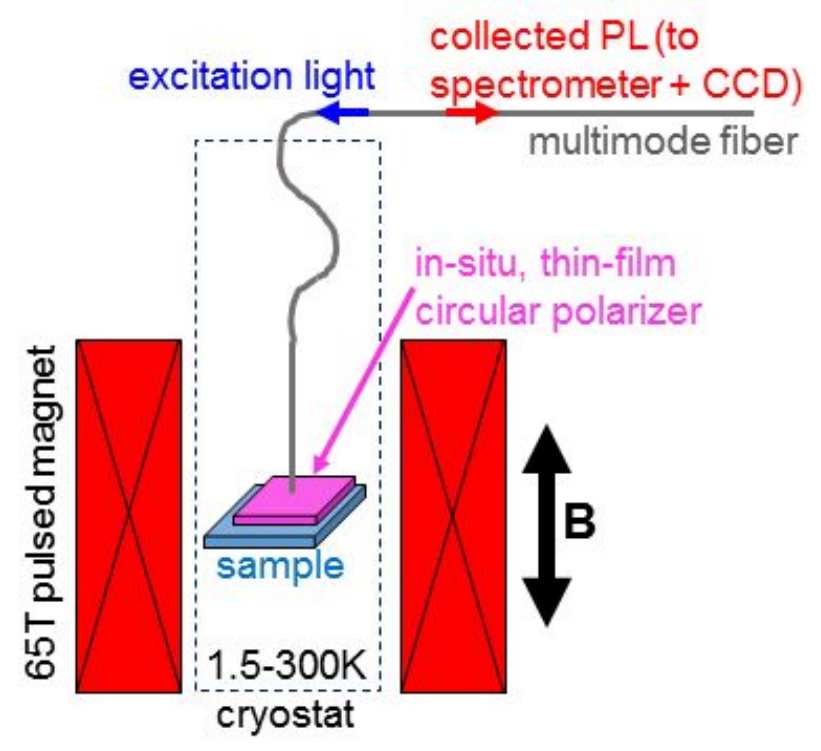

Supporting Figure S4 -The NCs samples are mounted onto a custom fiber-coupled probe residing in a He-bath cryostat with a long tail extending into the bore of a $65 \mathrm{~T}$-class pulsed magnet. Pump laser light is directed towards the sample using a $600-\mu \mathrm{m}$ diameter multimode optical fiber. The same fiber is used to collect the emitted PL. Thin film circular polarizers were used to select $\sigma^{-}$and $\sigma^{+}$polarized PL. Full optical spectra are acquired every $1 \mathrm{~ms}$ continuously throughout the magnet pulse ( $\sim 50 \mathrm{~ms}$ duration) using a fast CCD camera. To switch between different circular polarizations, we switched current direction in the magnet. 


\section{Supporting Figure S5 -Schematic depiction of the 15T magneto-optical experiment.}

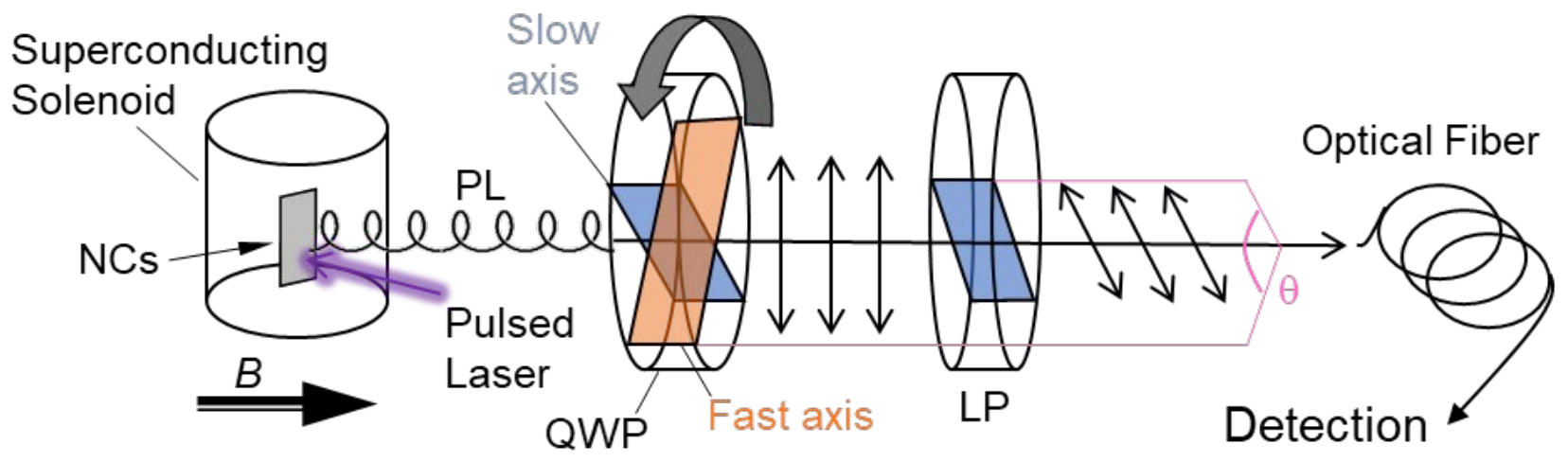

Supporting Figure S5 - Schematic of the circular-polarization resolves magneto-PL setup used in the 15T experiments. A film of DiB NCs dip-coated onto a glass substrate is mounted in a single-coil superconducting solenoid with direct optical access and is excited with a pulsed ultraviolet laser (photon energy is $3.06 \mathrm{eV}$ ). The emitted two-color light is resolved for its handedness by the quarter wave plate (QWP) and the linear polarizer (LP) mounted before a light-collecting input of an optical fiber coupled to a $0.55 \mathrm{~m}$ spectrometer and a chargecoupled-device detector. Right- and left-handed photons are selected by adjusting the angle $\theta$ between the fast axis of the QWP and the optical axis of the LP. 
Supporting Figure S6 - Circular-polarization-resolved PL decays and time-dependent $\boldsymbol{P}_{\mathrm{c}}$.
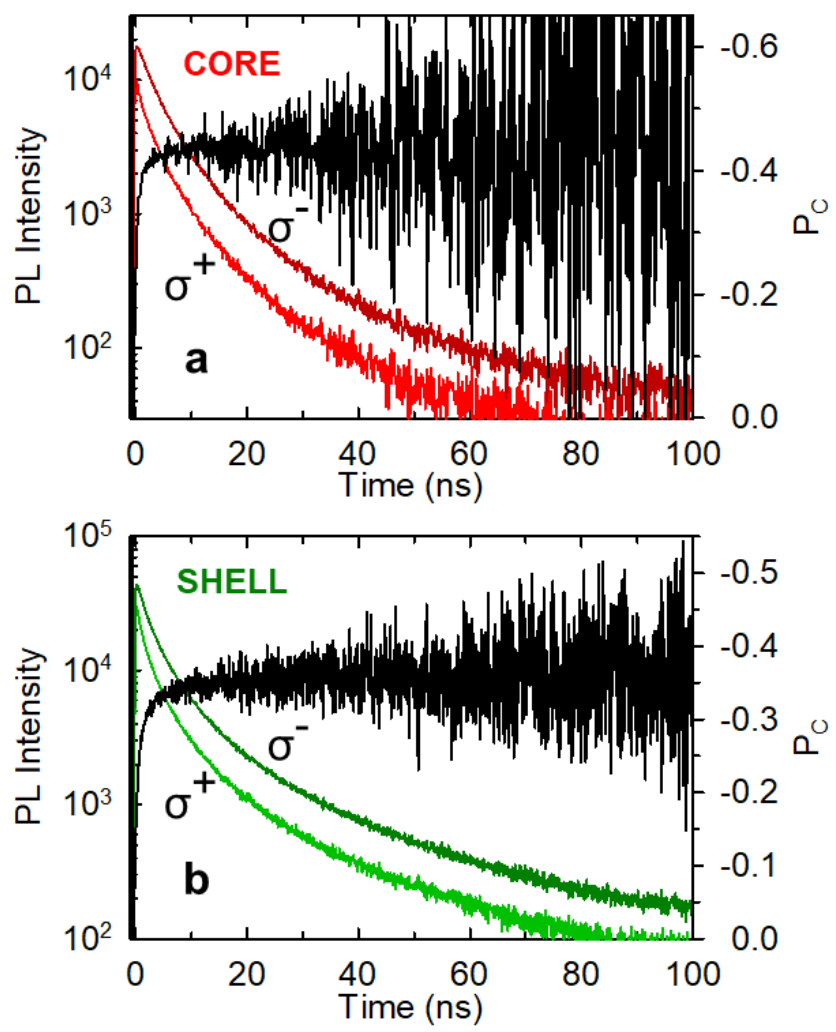

Supporting Figure S6 - (a) Core and (b) shell CP-resolved PL decays acquired at $B=15 \mathrm{~T}$ and $T=4.2$ K. Dark red and green curves refer to left-handed circularly polarized PL decays, whereas light red and green curves refer to right-handed circularly polarized PL decays. The black curves are the respective time-dependent $P_{\mathrm{c}}$ values, calculated using Equation (1). 
Supporting Figure S7 - Complete set of time-dependent $\boldsymbol{P}_{\mathrm{c}}$ data in different magnetic fields.
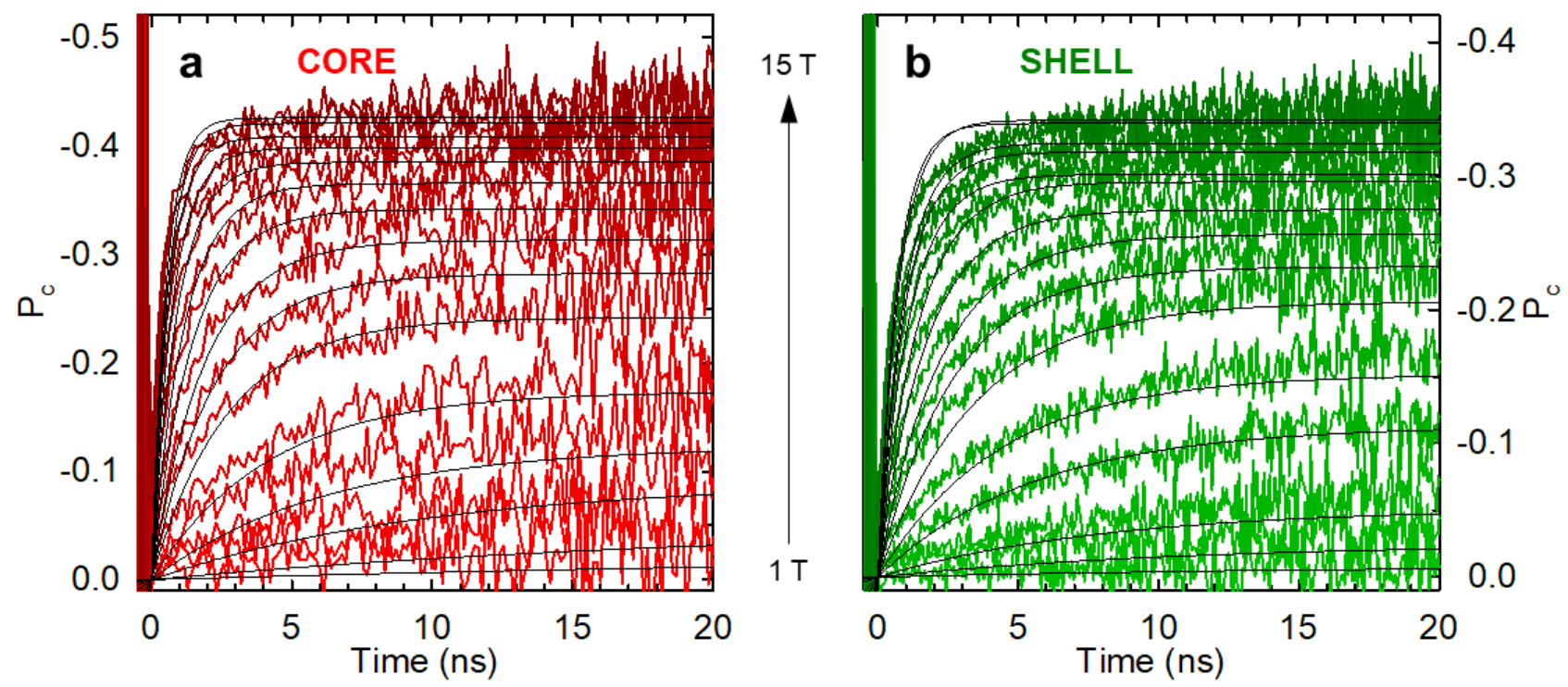

Supporting Figure S7 -Complete sets of time-resolved $P_{\mathrm{c}}$ curves for core (a) and shell (b) emissions for $B$ increasing from $1 \mathrm{~T}$ to $15 \mathrm{~T}$ with $1 \mathrm{~T}$ steps. The black curves are the results of fitting with Equation (2). 
Supporting Figure S8 - Time-dependent $P_{c}$ data at different temperatures $(B=5 T)$.

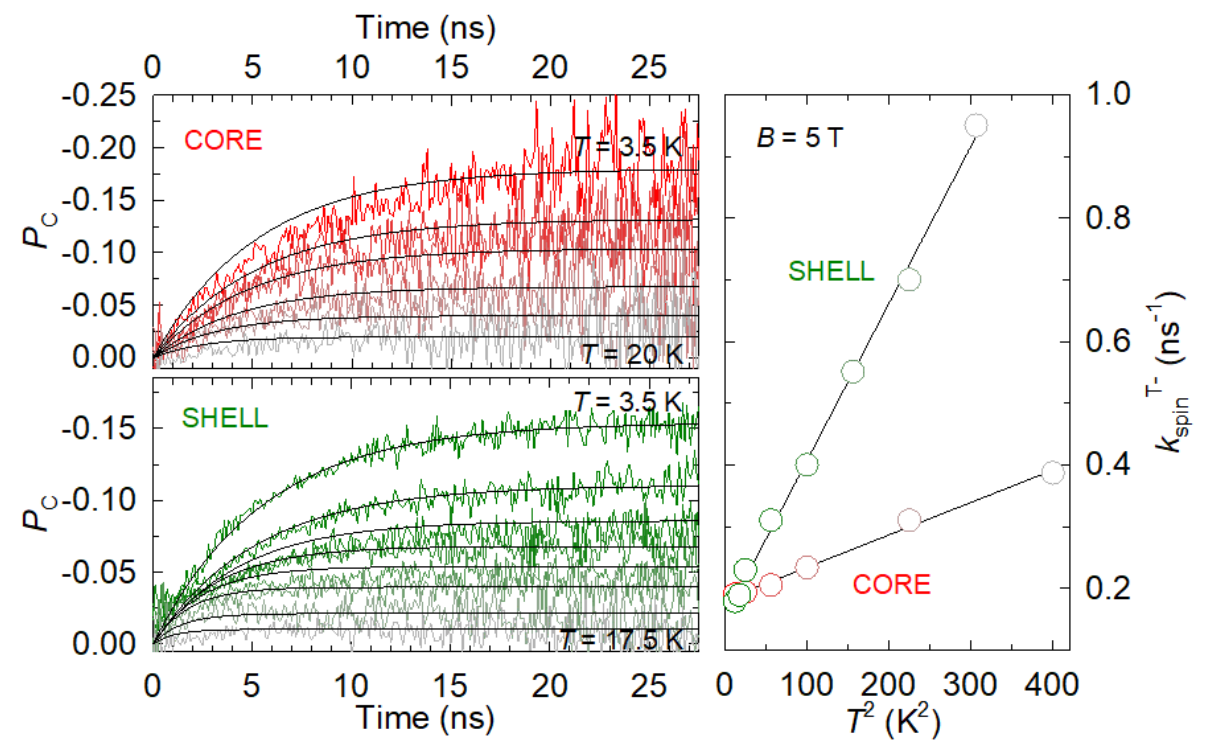

Supporting Figure S8 - Left panels: Complete sets of time-resolved $P_{\mathrm{c}}$ curves for core (top) and shell (bottom) emissions for $T$ increasing from $3.5 \mathrm{~K}$ to $20 \mathrm{~K}(B=5 \mathrm{~T})$. The black curves are the results of fitting with Equation (2). Right panel: Spin-flip rate values extracted from the $P_{\mathrm{C}}$ dynamics as a function of $T^{2}$. 
Supporting Table S1 - Parameters obtained by fitting $k_{\text {spin }}$ versus $B$ with Equation (3) (T= 4.2 K).

\begin{tabular}{c|c|c} 
& CORE & SHELL \\
\hline$k_{\text {spin }, 0}^{T-}\left[\mathrm{ns}^{-1}\right]$ & $2 \times 10^{-2}$ & $5 \times 10^{-2}$ \\
\hline$\alpha\left[\mathrm{ns}^{-1} \mathrm{~T}^{-2}\right]$ & $6.9 \times 10^{-3}$ & $5.6 \times 10^{-3}$
\end{tabular}

Supporting Table S2 - Parameters obtained by fitting $P_{c}^{e q}$ and $P_{c}^{\text {int }}$ versus $B$ with Equations (5) and (7), respectively.

\begin{tabular}{c|c|c} 
& CORE & SHELL \\
\hline hole $g$-factor & -0.85 & -1.2 \\
\hline$i$ & 0.245 & 0.185 \\
\hline$f$ & 0.255 & 0.195
\end{tabular}

\section{References}

1. Galland, C.; Brovelli, S.; Bae, W. K.; Padilha, L. A.; Meinardi, F.; Klimov, V. I.; Nano Lett. 2013, 13, (1), 321-328.

2. $\quad$ Yang, Y. A.; Wu, H.; Williams, K. R.; Cao, Y. C.; Angew. Chem. 2005, 117, (41), 6870-6873. 